\title{
Complex dynamics in a one-block model for earthquakes
}

\author{
Raúl Montagne and G. L. Vasconcelos* \\ Laboratório de Física Teórica e Computacional, Departamento de Física, \\ Universidade Federal de Pernambuco, 50670-901, Recife, Brazil.
}

\begin{abstract}
A two-dimensional earthquake model that consists of a single block resting upon a slowly moving rough surface and connected by two springs to rigid supports is studied. Depending on the elastic anisotropy and the friction force three generic regimes are possible: i) pure creep; ii) pure stick-slip motion; and iii) a mixed regime. In all cases the long-time dynamics (fixed point, periodic orbit or chaos) is determined by the direction of the pulling velocity. The possible relevance of our findings to real faults is briefly discussed.
\end{abstract}

Key words: Earthquake models, Nonlinear dynamics, Creep, Stick-slip PACS: 91.30.-f, 05.45.-a, 62.20.-x

\section{Introduction}

Since the seminal work of Burridge and Knopoff [1], spring-block models have been recognized as useful tools to study earthquake dynamics [2]. Because they are simple to treat, both theoretically and computationally, spring-block models can provide important physical insights that might otherwise be much more difficult to obtain. Models with many blocks have been used to investigate the origin of certain power laws that appear in the statistics of earthquakes, such as the Gutenberg-Righter law for the size-distribution of earthquakes [2]. Spring-block models with only a few degrees of freedom, on the other hand, can probe the basic dynamics of frictional sliding and are also of interest in their own right as examples of low-dimensional nonlinear dynamical systems.

\footnotetext{
* Corresponding author. Tel.: +55-81-3271-8450; fax: +55-81-3271-0359

Email addresses: montagne@lftc.ufpe.br (Raúl Montagne), giovani@lftc.ufpe.br (G. L. Vasconcelos).
} 
For instance, two-block models have been found to exhibit chaos [3,4,5], thus suggesting that actual faulting might perhaps be a chaotic phenomena. Even in the simplest case of a one-block model (with the block moving on a line) the dynamics is highly nontrivial. Indeed, this system displays a discontinuous transition from creep to stick-slip motion as one varies a parameter governing the dependency of the friction force on the velocity [6]. A two-dimensional version of the one-block model was recently considered [7] and a richer dynamics was found, including a chaotic regime where stick-slip events occur intermittently amidst creep.

In this paper we present a detailed study of a $2 \mathrm{D}$ one-block model for earthquakes, in which the block rests upon a moving planar surface and is connected by springs to two perpendicular rigid walls, with a velocity-weakening friction force acting between the slider and the surface. An earlier discussion on this model was reported by Ryabov and Ito [7]. Here we give a much more complete analysis and present several novel and surprising results. We show that in the limit of very slow pulling the model is governed by three dimensionless parameters, namely, the asymmetry parameter $\kappa$ corresponding to the ratio between the two spring constants, a parameter $\gamma$ governing the decrease of friction with velocity, and the direction $\theta_{\nu}$ of the pulling velocity. Depending on the values of $\kappa$ and $\gamma$, the model displays three possible generic regimes: i) pure creep; ii) pure stick-slip motion; and iii) a mixed regime where both creep and stick-slip occur. In each of these cases, the long-time dynamics (fixed point, periodic orbit or chaos) is starkly dependent on $\theta_{\nu}$, as we will see below.

\section{The model}

The model we consider consists of a block of mass $m$ connected to motionless walls by two springs of stiffness $k_{x}$ and $k_{y}$, as shown in Fig. 1a. The position of the block is described in a system of coordinates $(x, y)$ fixed to the spring supports, with the origin being placed at the point where the elastic force vanishes. The block rests upon a surface that moves with a constant velocity $\vec{V}$, where $|\vec{V}| \ll 1$. There is friction between the block and the moving surface, so that initially the block moves with the substrate until the elastic force $\vec{F}_{\text {el }}$ overcomes the static friction force $F_{0}$, at which point the block slips with respect to the surface. In the slip phase of the motion, we adopt a commonly used velocity-weakening friction law [8], in which the magnitude of the friction

force $\vec{F}_{\text {fr }}$ is given by $\left|\vec{F}_{\mathrm{fr}}\right|=F_{0} \Phi\left(v_{\mathrm{r}} / V_{\mathrm{f}}\right)$, where $v_{\mathrm{r}}=\sqrt{\left(\dot{x}-V_{x}\right)^{2}+\left(\dot{y}-V_{y}\right)^{2}}$ is the relative velocity of the slider with respect to the surface, $V_{\mathrm{f}}$ is a typical velocity scale for the friction force, and $\Phi(x)=1 /(1+x)$.

In the remainder of the paper we work with non-dimensional quantities, where 

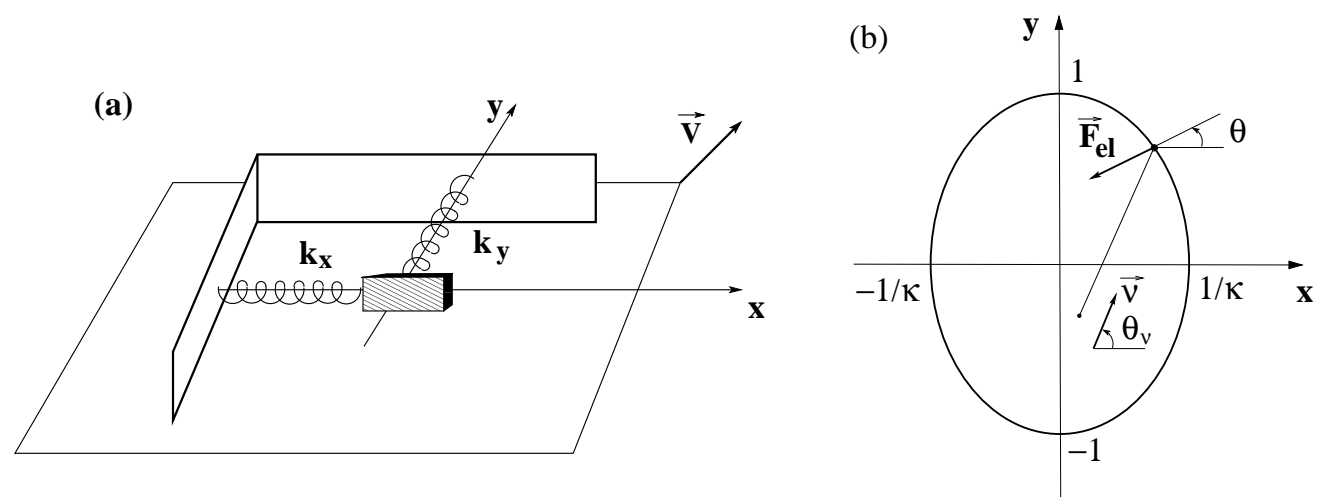

Fig. 1. (a) Two-dimensional spring-block model and (b) the ellipse $C=\left\{(x, y) \mid \kappa^{2} x^{2}+y^{2}=1\right\}$ where the elastic force equals the static friction. Starting in a point inside $C$, the block is dragged by the surface along the direction $\theta_{\nu}$ of the pulling velocity until it hits a point on $C$, whereupon slip begins. The angle $\theta$ denotes the direction opposite to the elastic force at this point.

we have rescaled the length and time scales by $F_{0} / k_{y}$ and $\sqrt{m / k_{y}}$, respectively, and velocities by $F_{0} / \sqrt{m k_{y}}$. In these units, the dimensionless parameters characterizing the problem are the pulling velocity $\vec{\nu}$, the anisotropy parameter $\kappa=k_{x} / k_{y}$, and the friction parameter $\gamma=1 / 2 \nu_{\mathrm{f}}$, where $\nu_{\mathrm{f}}$ is the dimensionless characteristic velocity of the friction. If we now denote by $C$ the curve of maximum displacement, where the condition $\left|\vec{F}_{\text {el }}\right|=F_{0}$ is verified, it then follows that $C$ corresponds (in dimensionless units) to the ellipse $\kappa^{2} x^{2}+y^{2}=1$; see Fig. 1b. Starting from relative rest at a point inside $C$, the block will be dragged by the surface until it reaches a point on $C$, upon which the block starts to slide. In the slip phase the equations of motion are given by

$$
\begin{aligned}
& \ddot{x}=-\kappa x+\Phi\left(2 \gamma v_{\mathrm{r}}\right) \cos \varphi_{\mathrm{fr}}, \\
& \ddot{y}=-y+\Phi\left(2 \gamma v_{\mathrm{r}}\right) \sin \varphi_{\mathrm{fr}},
\end{aligned}
$$

where the angle $\varphi_{\text {fr }}$ gives the direction of the friction force. These equations represent two coupled (nonlinear) oscillators, and in this sense the system is somewhat similar to the two-block model considered in Ref. [3]. Our 2D one-block model is, however, qualitatively richer in that it contains a new parameter, namely, the direction of the pulling velocity which strongly affects the dynamics of the system, as we will see shortly.

A final caveat about the model concerns the choice of direction of the friction force. The problem comes about because at the onset of motion the friction force is opposite to the elastic force, whereas during the slip phase of the motion the friction force is expected to point in the direction opposite to the relative velocity. Since the elastic force is not in general collinear with the velocity, we should thus prescribe a mechanism that allows the switching of direction of the friction force. Here we follow Ryabov and Ito [7] and assume 
that in the acceleration phase of the block motion, the direction $\varphi_{f r}$ of the friction force is chosen according to the following rule

$$
\varphi_{f r}=\pi+\varphi_{e l}-\left(\varphi_{e l}-\varphi_{v r}\right)\left[1-\exp \left(-\Omega v_{v r}\right)\right]
$$

where $\varphi_{v r}$ and $\varphi_{e l}$ denote the directions of the relative velocity and the elastic force, respectively, and $\Omega$ is a parameter describing the 'memory' of the friction force. (The qualitative behavior of the model does not depend on the value of $\Omega$ and so we have used $\Omega=50$ throughout the paper.) In the deceleration phase, the friction force is kept opposite to the velocity to ensure that the block always comes to a stop.

The dynamics of the model described above can be conveniently described in terms of a one-dimensional map. To see this, let us first parametrize the curve $C$ in terms of the angle $\theta$ defined by the direction opposite to the elastic force with respect to the $x$ axis; see Fig. 1b. As a function of $\theta$, the coordinates $\left(x_{0}, y_{0}\right)$ of a point on $C$ are given by the following parametric equations: $x_{0}=$ $\kappa^{-1} \cos \theta, y_{0}=\sin \theta$. Now suppose that the block starts to slip from a given point on $C$ labeled by $\theta$. The block will slide for a while until it eventually comes to a stop with respect to the moving surface. After this, the block sticks to the surface and is brought back to another point $\theta^{\prime}$ on $C$, where a new slip cycle begins, and so on. Thus, the overall dynamics of the model can be described by a one-dimensional map $\theta^{\prime}=f(\theta)$. Obviously the mapping function $f(\theta)$ cannot be computed explicitly since this entails solving (1) and (2) during the slip phase of the motion. However, for a given set of parameters $\kappa, \gamma$, and $\vec{\nu}$, the map $f(\theta)$ can be easily constructed on the computer [7].

\section{Analysis of the model and results}

We begin the analysis of our model by considering the early stages of a slip event. Suppose that the block, as it is being dragged by the moving surface, reaches a point $\left(x_{0}, y_{0}\right) \in C$ where relative motion begins. At the onset of slip the block will move in the direction of the elastic force $\vec{F}_{\text {el }}=\left(-\kappa x_{0},-y_{0}\right)$, while the friction force will act in the opposite direction. In order to study the nature of the ensuing motion, we linearize the equations of motion in the directions parallel and perpendicular to the elastic force. To accomplish this, it is convenient first to switch to the reference frame where the substrate is at rest and then write the equations of motion in the coordinates $(\xi, \eta)$, defined as the block displacement in the directions parallel and perpendicular to the elastic force, respectively. The resulting linearized equations are

$$
\ddot{\xi}=-A \xi+\kappa(\kappa-1) x_{0} y_{0} \eta+2 \gamma \dot{\xi}+\alpha t
$$



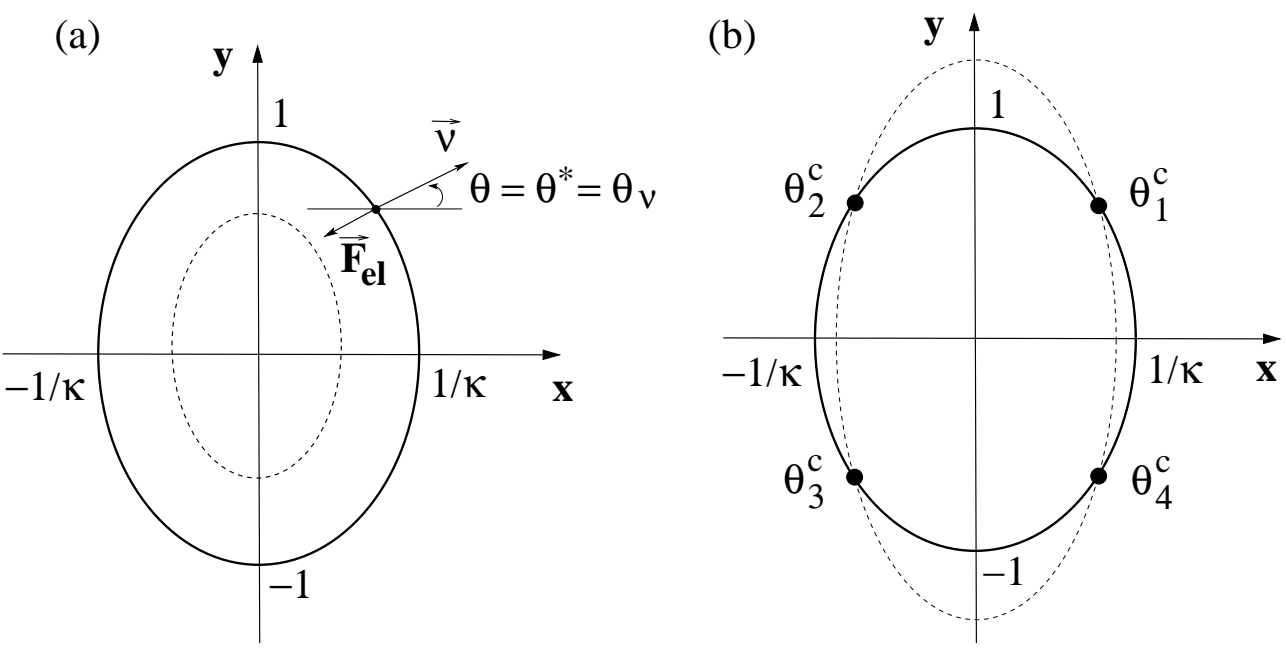

Fig. 2. The ellipse $C$ of maximum displacement (solid line) and the critical ellipse (dashed line). We have $\gamma^{2}<1$ in (a) and $1<\gamma^{2}<\kappa$ in (b).

$$
\ddot{\eta}=\kappa(\kappa-1) x_{0} y_{0} \xi-\kappa\left(\kappa x_{0}^{2}+y_{0}^{2}\right) \eta+\beta t,
$$

where

$$
A=\kappa^{3} x_{0}^{2}+y_{0}^{2}, \quad \alpha=-\left(\kappa^{2} x_{0} \nu_{x}+y_{0} \nu_{y}\right), \quad \beta=\kappa\left(y_{0} \nu_{x}-y_{0} \nu_{y}\right) .
$$

We see from (5) that in the limit $\nu \rightarrow 0$ the transversal coordinate $\eta$ can grow only if so does the component $\xi$. We can thus set $\eta=0$ in (4), so that the linear equation for the variable $\xi$ becomes

$$
\ddot{\xi}-2 \gamma \dot{\xi}+A \xi=\alpha t .
$$

Equation (7) for the case $A=1$ was studied in great detail by one of the present authors [6]. There, it was shown that in the limit that $\nu \rightarrow 0$ the system undergoes a phase transition at $\gamma=1$ in the following sense: for $\gamma<1$ there is only creep, meaning that the linear approximation is always valid and the amplitude of a slip event vanishes as $\nu \rightarrow 0$, whereas for $\gamma>1$ one has stick-slip motion in which case the slip amplitude remains finite as $\nu \rightarrow 0$. These results can be trivially extended to the general case given in (7). Here the critical point is reached when the condition $\gamma^{2}=A$ is satisfied, so that if $\gamma^{2}<A$ we have creep, while for $\gamma^{2}>A$ stick-slip occurs. Note, however, that since the parameter $A$ depends on the point $\left(x_{0}, y_{0}\right)$ where the slip was initiated, it follows that for a critical point to exist the ellipse defined by the critical condition $A=\gamma^{2}$ must intersect the curve $C$. If such an intersection occurs, then the model displays both creep and stick-slip, otherwise the motion is either pure creep or pure stick-slip, as described next.

Pure creep: $\gamma<1$. In this case the critical ellipse $A=\gamma^{2}$ is inside $C$ (Fig. 2a), so that $\gamma^{2}<A$ for any point $\left(x_{0}, y_{0}\right) \in C$, and hence the linear approximation 

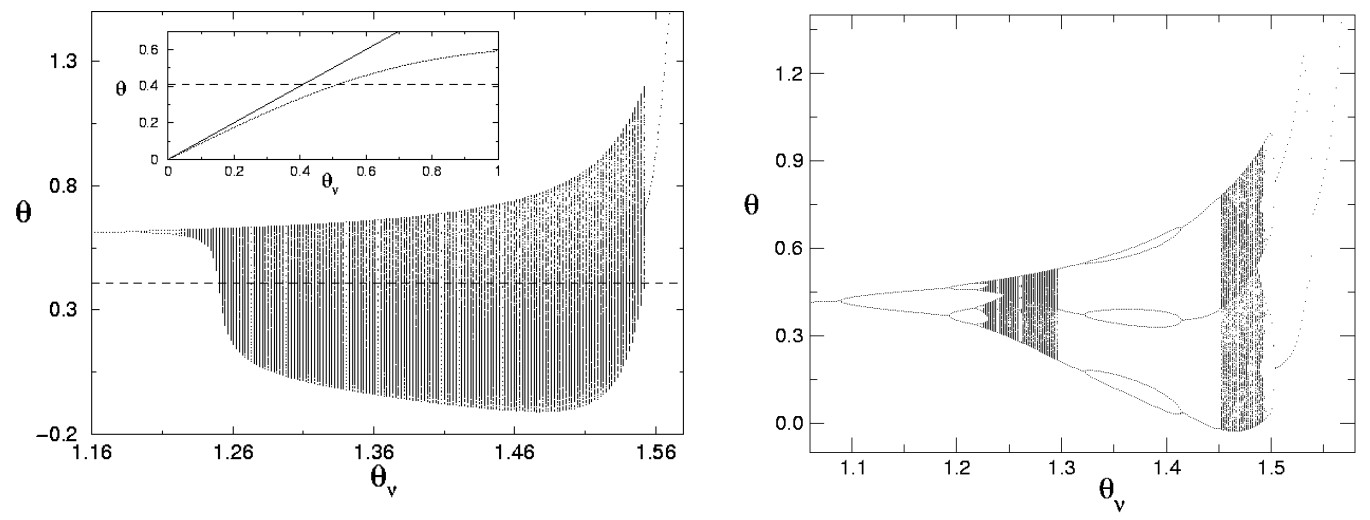

Fig. 3. Bifurcation diagram for the map $f(\theta)$ in terms of the control parameter $\theta_{\nu}$. In (a) we have $\gamma=6.5, \kappa=50, \nu=10^{-4}$, corresponding to the creep and stick-slip regime, and the dashed line denotes the critical point $\theta_{c}=0.41$. The inset shows the initial part of the bifurcation diagram, with the solid straight line representing the $45^{\circ}$ line. In (b) we have stick-slip only with $\gamma=6.5, \kappa=18.95$, and $\nu=10^{-3}$.

(7) always holds (in the limit $\nu \rightarrow 0$ ). The long-time dynamics of the system is quite simple and can be easily described in terms of the map $\theta^{\prime}=f(\theta)$ introduced above. Let us define by $\theta_{\nu}$ the direction of the pulling velocity. Then for any initial condition the block will reach the fixed point $\theta=\theta^{*}=\theta_{\nu}$, where the elastic force is opposite to $\vec{\nu}$ (see Fig. 2a), so that the resulting motion is essentially one-dimensional creep: starting from the point on $C$ labeled by $\theta^{*}$, the block slides a very small distance along the direction of the elastic force, stops, and then is brought back to the same point $\theta^{*}$, and so on.

Creep and stick-slip: $1<\gamma^{2}<\kappa$. In this situation the critical ellipse and $C$ intersect each other at four critical points $\theta_{i}^{c}$, where $\tan ^{2} \theta_{i}^{c}=\frac{\kappa-\gamma^{2}}{\gamma^{2}-1}$; see Fig. 2b. Without loss of generality, let us assume that $\theta_{\nu}$ is restricted to the first quadrant, so that for $0 \leq \theta<\theta_{1}^{c}$ we have creep $\left(\gamma^{2}<A\right)$, whereas for $\theta_{1}^{c}<\theta \leq \pi / 2$ stick-slip occurs $\left(\gamma^{2}>A\right)$. Thus, for $\theta_{\nu} \in\left[0, \theta_{c}\right)$ the creep fixed-point $\theta=\theta^{*}=\theta_{\nu}$ is the only attractor. This is illustrated in the inset of Fig. 3a, where we plot the initial part of the bifurcation diagram for the map $f(\theta)$ with $\gamma=6.5, \kappa=50, \nu=10^{-4}$, and $\theta_{\nu}$ as the control parameter. In this inset, the dashed line represents the critical point $\theta_{c}=0.41$ and we see that for $\theta_{\nu}<\theta_{c}$ the fixed point indeed follows closely the $45^{\circ}$ line (solid straight line), with the small deviation being caused by the finite value of $\nu$ that smoothes out the transition from creep to stick-slip. As $\theta_{\nu}$ increases past $\theta_{c}$, the system remains in a stick-slip fixed point for awhile but eventually undergoes a sequence of period-doubling bifurcations leading to chaos, as seen in the main bifurcation diagram shown in Fig. 3a. For larger $\theta_{\nu}$, the system behaves intermittently [7] in the following sense: the block spends a long time in the creep region (below the dashed line) until it eventually crosses the critical point $\theta_{c}$, after which it undergoes a large slip event and is reinjected back into the creep region, and so on. Finally, as $\theta_{\nu}$ approaches $\pi / 2$ there is a reverse period-doubling cascade back to a fixed point. 


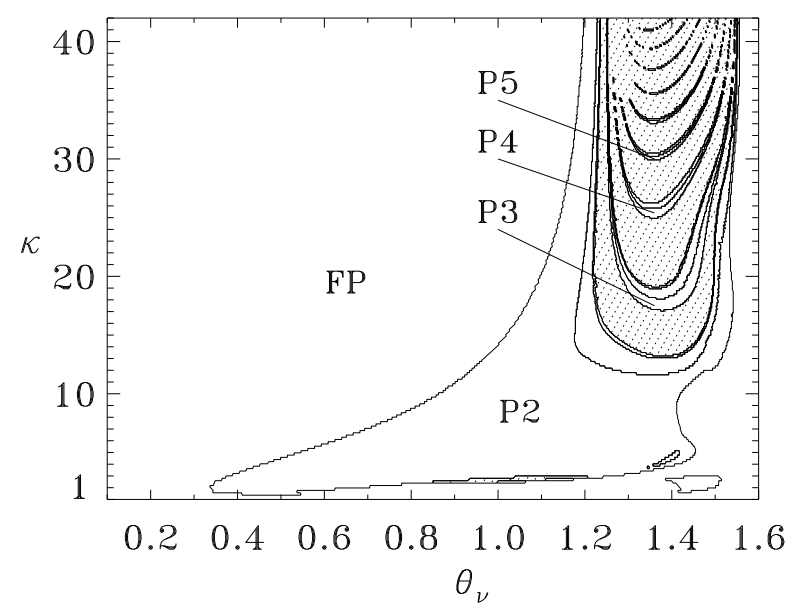

Fig. 4. Phase diagram in the plane $\left(\theta_{\nu}, \kappa\right)$ for the pure stick-slip regime. $F P$ denotes the region with a stable fixed point, $P n$ indicates regions of period- $n$ orbits, and the shaded areas represent chaotic regions. Here we used $\gamma=6.5$ and $\nu=10^{-3}$.

Pure stick-slip: $\gamma^{2}>\kappa$. Here the critical ellipse is exterior to $C$ and so we have $\gamma^{2}>A$ for any point on $C$, hence the block always undergoes stick-slip motion. As the parameters $\kappa$ and $\theta_{\nu}$ are varied (with $\gamma$ fixed), the system now displays a very rich behavior that is summarized in the phase diagram shown in Fig. 4, whose main features we shall briefly discuss. First we see that for small values of $\theta_{\nu}$ the system always reaches a stable fixed point (region labeled by $F P$ ), where slip events occur periodically. As $\theta_{\nu}$ increases (with $\kappa$ fixed), what follows next depends on the value of $\kappa$. For $\kappa$ very close to 1 the fixed point remains stable for all values of $\theta_{\nu}$; see bottom of Fig. 4. However, for larger $\kappa$ the fixed point eventually goes unstable and a orbit of period two is born (region P2). The fate of this period-2 orbit, as $\theta_{\nu}$ increases further, also depends on the value of $\kappa$. For small $\kappa$, e.g., $\kappa=10$, the system simply undergoes a reverse period-doubling bifurcation back to the fixed point. For intermediate values of $\kappa$, say, $\kappa=16$, one observes a full period-doubling cascade leading to chaos (shaded region), followed by a reverse cascade. For larger $\kappa$, windows of periodic orbits and their associated cascades appear inside the chaotic region. Several of these windows are clearly visible in Fig. 4, although we have labeled only the first three of them, namely, the windows of period 3, 4, and 5 . Note also that depending on the value of $\kappa$ the secondary period-doubling cascades may not develop fully. An example of this is given in Fig. 3b where we show the bifurcation diagram for the map $f(\theta)$ with $\gamma=6.5$ and $\kappa=18.95$. 


\section{Conclusions}

In conclusion, we have studied a two-dimensional one-block model for earthquakes that shows a very rich behavior and is able to reproduce a host of relevant dynamical regimes such as creep, stick-slip (periodic and chaotic) and intermittent stick-slip amidst creep. Particularly interesting is our finding

that the dynamics of the model is strongly dependent on the direction of the pulling velocity. This property thus suggests that, in addition to the friction force acting on a fault and the stiffness of the loading system, the direction of shear might also play an important role in determining the fault seismic activity (or lack thereof). Such mechanism could perhaps help explaining, for instance, why different segments of a fault system may exhibit distinct seismic patterns although they presumably have similar geophysical properties. These are interesting possibilities that certainly deserve to be investigated further.

\section{Acknowledgements}

We thank M. A. F. Gomes for a critical reading of the manuscript. Financial support from the Brazilian agencies CNPq and FINEP and from the special research programs PRONEX and CTPETRO is acknowledged. R. M. acknowledges financial support from C.S.I.C. (Uruguay) and the Programa de Desarrollo de Ciencias Básicas (PEDECIBA, Uruguay).

\section{References}

[1] R. Burridge, L. Knopoff, Seism. Soc. Am. Bull 57 (1967) 341.

[2] For a recent review on spring-block models, see, e.g., D. L. Turcotte, Rep. Prog. Phys. 62 (1999) 1377.

[3] J. Huang, D. L. Turcotte, Nature 348 (1990) 234.

[4] V. B. Ryabov, K. Ito, Phys. Rev. E 52 (1995) 6101.

[5] M. de Sousa Vieira, Phys. Rev. Lett. 82 (1999) 201.

[6] G. L. Vasconcelos, Phys. Rev. Lett. 76 (1996) 4865.

[7] V. B. Ryabov, K. Ito, Pure appl. Geophys. 158 (2001) 919.

[8] M. de Sousa Vieira, G. L. Vasconcelos, S. Nagel, Phys. Rev. E 47 (1993) R2221. 